\title{
Expression of Barh/2 and its relationship with Pax6 expression in the forebrain of the mouse embryo
}

\author{
Elisa V. Parish, John O. Mason and David J. Price*
}

\begin{abstract}
Background: The transcription factor Barhl2 is an antiproneural transcription factor with roles in neuronal differentiation. The functions of its homologue in Drosophila development are better understood than its functions in mammalian brain development. Existing evidence suggests that its expression in the embryonic forebrain of the mouse is regional and may complement that of another transcription factor that is important for forebrain development, Pax6. The aim of this study is to provide a more detailed description of the Barh/2 expression pattern in the embryonic forebrain than is currently available, to relate its expression domains to those of Pax 6 and to examine the effects of Pax 6 loss on Barh/2 expression.

Results: We found that Barh/2 is expressed in the developing diencephalon from the time of anterior neural tube closure. Its expression initially overlaps that of Pax6 in a central region of the alar diencephalon but over the following days their domains of expression become complementary in most forebrain regions. The exceptions are the thalamus and pretectum, where countergradients of Pax6 and Barh/2 expression are established by embryonic day 12.5, before overall Pax6 levels in these regions decline greatly while Barh/2 levels remain relatively high. We found that Barh/2 expression becomes upregulated in specifically the thalamus and pretectum in Pax6-null mice.
\end{abstract}

Conclusions: The region-specific expression pattern of Barh 12 makes it likely to be an important player in the development of region-specific differences in embryonic mouse forebrain. Repression of its expression in the thalamus and pretectum by Pax6 may be crucial for allowing proneural factors to promote normal neuronal differentiation in this region.

Keywords: Mouse, Development, Thalamus, Forebrain, Gene expression, Zona limitans intrathalamica, Pax6, Barhl2

\section{Background}

The development of the central nervous system depends on the actions and interactions of transcription factors and morphogens linked together in complex gene regulatory networks. These networks serve to finely control processes such as tissue patterning and neuronal subtype specification $[1,2]$. The bar homeobox-like (Barhl) family of transcription factors, Barhl1 and Barhl2, are the mammalian homologues of the Drosophila bar homeobox (BarH) transcription factors BarH1 and BarH2 [3]. Barhl2 is strongly expressed in the proliferative zones

\footnotetext{
*Correspondence: david.price@ed.ac.uk Centre for Integrative Physiology, The University of Edinburgh, Hugh Robson Building, Edinburgh EH8 9XD, UK
}

of specific regions in the mammalian forebrain [4]. Its interactions with the many other transcription factors expressed in these regions are likely to be critical for normal forebrain development.

The proteins encoded by bar genes and their homologues in other species are characterised by the presence of a homeodomain along with either one or two FIL domains-DNA-binding regions that are rich in the amino acids phenylalanine $(\mathrm{F})$, isoleucine $(\mathrm{I})$, and leucine (L) [5]. Transcription factors containing FIL domains can act as transcriptional repressors [6] via a mechanism involving their recruitment of the Drosophila co-repressor Groucho or its homologues in other species [7-13]. The Drosophila BarH genes are known to prevent ectopic 
neurogenesis in the fly retina by inhibiting the expression of atonal (ato) [14], a proneural transcription factor featuring a basic helix-loop-helix (bHLH) motif. There is evidence that the actions of the mammalian Barhl genes are mediated at least in part by their regulation of atonalrelated bHLH transcription factors, such as those of the Neurogenin (Ngn) family [5, 15, 16].

Barhl2 plays roles in neuronal subtype specification in the vertebrate nervous system. In the retina, Barhl2 is required for amacrine cell (AC) subtype specification. Loss of Barhl2 leads to the specification of increased numbers of cholinergic ACs at the expense of glycinergic and GABAergic ACs [17], while the premature expression of Barhl2 in the zebrafish retina induces the differentiation of GABAergic ACs at the expense of nonGABAergic ACs and photoreceptors [18]. In the mouse spinal cord Barhl2 serves to specify dl1 interneuron subtype, and the loss of Barhl2 leads to an increase in the number of contralaterally-projecting interneurons, with a reduction in the number that project ipsilaterally [19].

Studies in Xenopus have shown that the Xenopus BarH2 homologue, Xbarhl2 [5], promotes the formation of the zona limitans intrathalamica (ZLI) [20], a forebrain organizer region that patterns the diencephalon via the secretion of morphogens including Sonic hedgehog (Shh) [21, 22]. Another transcription factor, paired-box 6 (Pax6), has an opposite effect on the ZLI, limiting its size [22-24]. Published data on Barhl2 expression, which is limited, suggests that it might complement that of Pax6 throughout much of the embryonic mouse forebrain with the possible exception of the thalamic ventricular zone, in which both genes appear to be strongly expressed at some embryonic stages [4, 25-27]. We carried out a comprehensive analysis of the forebrain expression of Barhl2 in embryonic mice at a range of developmental stages, using qualitative and quantitative methods to examine its relationship with the expression of Pax6. We examined expression of Barhl2 in the Pax6-null mutant mouse to test for a functional relationship between the expression patterns of Barhl2 and Pax6.

\section{Methods}

\section{Experimental animals and ethics statement}

All experimental work was carried out in accordance with the UK Animals (Scientific Procedures) Act 1986 and UK Home Office guidelines [28]. All protocols were reviewed and approved by the named veterinary surgeons of the College of Medicine and Veterinary Medicine, the University of Edinburgh, prior to the commencement of experimental work.

Wild-type mice used were of the Mus musculus strain CD-1 ${ }^{\circledR}$ [29]. Timed matings were set up between CD-1 ${ }^{\circledR}$ males and females. The day on which a vaginal plug could be observed was taken to be embryonic day 0.5 (E0.5). Embryos were harvested at E8.5-E13.5. Pax6-null mice used were of the Mus musculus strain Sey ${ }^{E d}$ [30]. Crosses were set up between $\mathrm{Pax}^{+/ S e y}$ males and $\mathrm{Pax}^{+/ \mathrm{Sey}}$ females to generate litters comprising $\mathrm{Pax}^{+/+}, \mathrm{Pax}^{+/ \mathrm{Sey}}$, and $P a x 6^{\text {Sey/Sey }}$ embryos. Embryos were harvested at E11.5-E12.5. Pax $6^{\text {Sey/Sey }}$ embryos were identified by their lack of eyes.

\section{In situ hybridization}

Harvested embryos were fixed in a solution of $4 \%$ paraformaldehyde (Fisher Scientific) in phosphate buffered saline (PBS) (Oxoid) at $4{ }^{\circ} \mathrm{C}$ overnight before being sucrose-sunk as previously described [31] and fixed in a 1:1 mixture of $30 \%$ sucrose solution in PBS optimal cutting temperature (OCT) medium (Sakura). For chromogenic in situ hybridisation embryos were then cryosectioned at a thickness of $10 \mu \mathrm{m}$ before the protocol was performed as previously described [31]. For fluorescence in situ hybridization embryos were sectioned at a thickness of $16 \mu \mathrm{m}$ before the protocol was performed as previously described [32].

The RNA riboprobe for Pax6 was that described in Pinson et al. [33]. The RNA riboprobe for Barhl2 [4] was a kind gift from Asuka Suzuki-Hirano and Tomomi Shimogori. The Ngn2 probe was that described in Gradwohl et al. [34]. The Shh probe was that described in Echelard et al. [35].

\section{Immunohistochemistry}

Following fluorescence in situ hybridization for Barhl2, antigen retrieval was performed by microwaving sections in a $10 \mathrm{mM}$ aqueous solution of sodium citrate. Immunohistochemistry for Pax6 protein was performed as previously described [36]. The primary antibody used was rabbit Poly Pax6 (Covance Research Products) at a concentration of 1:400 in blocking solution. The secondary antibody used was goat anti-rabbit conjugated to AlexaFluor $488^{\circledR}$ (Abcam) at a concentration of 1:400 in blocking solution. Anti-Nestin primary antibody was used at 1:40 (Becton-Dickinson).

\section{Imaging}

Brightfield images were recorded with the Leica DMLB microscope and Leica Application Suite software. Fluorescence images were recorded using the Nikon A1RFLIM confocal microscope and Nikon Elements software. The "grab large image free shape" function of elements was used to compile a tiled image from several square images recorded at different regions of the tissue section. For each of the three channels (Pax6 immunostaining at $488 \mathrm{~nm}$, Barhl2 in situ hybridization staining with cyanine-3-tyramide at $554 \mathrm{~nm}$, DAPI staining of chromatin 
at $350 \mathrm{~nm}$ ) a 12-bit greyscale image was recorded at a resolution of $1028 \times 1028$ pixels. Each set of three greyscale images was saved as a stack. All confocal images were recorded at the Image Analysis MultiPhoton and Confocal Technologies (IMPACT) imaging facility, The University of Edinburgh.

\section{Quantification of image data}

The quantitative analysis was performed on images of coronal sections cut at the plane illustrated in Figs. 7 and 8A from each of three different embryos harvested at each of four developmental stages from E10.5 to E13.5 inclusive (12 embryos in total). The 12-bit greyscale images recorded for the Pax6 immunostaining channel $(488 \mathrm{~nm})$ and Barhl2 in situ hybridisation channel $(554 \mathrm{~nm})$ were analysed using the Fiji software package [37]. For each image, the segmented line tool was used to draw a line through the Pax6 and Barhl2-expressing progenitor populations, parallel with the ventricular surface of the diencephalon from the dorsal midline to the ZLI (Fig. 8A). The intensity plot profile tool was then used to obtain average pixel greyscale values (ranging from 0 to 4096) along the line. The process was carried out on both left and right sides of the brain and the average values at each position along the line were plotted against distance from the dorsal midline. For images of embryonic tissue harvested at E10.5-E12.5, the line was $40 \mu \mathrm{m}$ wide along its entire length. For embryos harvested at E13.5, the thickness of the neuroepithelium close to the dorsal midline had fallen below $40 \mu \mathrm{m}$, and so for this small region a $16 \mu \mathrm{m}$-wide line was used and intensity data from the two lines were subsequently combined. A linear regression trend line was calculated for each plot. The gradients of the trend lines for each of the three embryos analysed at each developmental stage were used to calculate the mean gradients of Pax6 and Barhl2 expression for each stage.

\section{Results}

Expression of Pax6 and Barh/2 in the embryonic forebrain We first used chromogenic in situ hybridization to examine the expression of Pax6 and Barhl2 separately, on adjacent coronal sections through a series of embryonic brains of increasing age. At E8.5 (Fig. 1A-F), neural tube closure is not yet complete and the two dorsal edges of the neural tube can be observed prior to their fusing to form the roofplate (double-headed arrow, Fig. 1A). At this stage Pax6 is expressed throughout most of the alar diencephalon and telencephalon (Fig. 1A-C) but is absent from their basal plate (Fig. 1A-C, summarized in Fig. 1M). Barhl2 is also expressed throughout much of the diencephalon, with strongest expression in alar regions overlapping the middle of the diencephalic domain of Pax6 expression (Fig. 1D-F, summarized in
Fig. 1M). Barhl2 expression is absent from the telencephalon (Fig. 1F).

At E9.5, following the closure of the neural tube and the formation of the roofplate (arrow, Fig. 1G), Pax6 continues to be strongly expressed throughout alar regions of the forebrain but not the basal plate (Fig. 1G-I). Barhl2 expression remains absent from the telencephalon (Fig. 1L, summarized in Fig. 1N). In the diencephalon, the Barhl2 expression domain has consolidated into a band of neuroepithelium running from ventral (in the basal plate: Fig. 1J) to dorsal (Fig. 1K, L), flanked by regions of diencephalic neuroepithelium expressing little or no Barhl2 (summarised in Fig. 1N). The alar component of this band overlaps a central strip of the diencephalic domain of $\mathrm{Pax} 6$ expression.

Previous work has shown that major regions of the diencephalon can be distinguished based on their morphology and their patterns of gene expression by E10.5. The ZLI is developing as a narrow Shh-expressing domain that spreads across the alar neural tube from ventral to dorsal, separating the prethalamus, which is rostral to the ZLI, from the thalamus, which is caudal to it $[21,38,39]$. Pax6 expression levels now show greater regional variation within the alar forebrain (Fig. 2A-C). It is strongly expressed in the pretectum, prethalamus and telencephalon but is absent from the ZLI (arrowheads, Fig. 2A). It is also absent from the mantle cells of the developing eminentia thalami (arrowhead, Fig. 2C), which link the diencephalon and the telencephalon on each side of the brain. In the thalamus, Pax6 expression levels are graded from low near the ZLI to high at the boundary with the pretectum (Fig. 2A-C). Strong Pax6 expression can also be seen in the retina of the developing eye (Fig. 2B). These expression patterns are summarized in Fig. 2M.

Unlike Pax6, Barhl2 is expressed within the developing ZLI itself at E10.5 (Fig. 2D). It is also expressed in the pretectum and throughout the majority of the thalamic ventricular zone, in the region of progenitor cells known as the pTh-C [22, 39, 40]. Barhl2 is not expressed in a narrow strip of progenitor cells immediately caudal to the ZLI, a region known as the pTh-R [22, 39, 40] (Fig. 2D). Barhl2 is also not expressed in the prethalamus (Fig. 2DF), where Pax6 expression is strong (Fig. 2A-C). In more rostral sections a domain of Barhl2 expression can be seen within the mantle zone of the developing eminentia thalami (arrowhead, Fig. 2F), where Pax6 is not expressed (Fig. 2C). Barhl2 expression remains absent from the eye and the telencephalon (Fig. 2E, F). These expression patterns are summarized in Fig. 2M: essentially, significant complementarity between the patterns of expression of these two genes is emerging at E10.5, with residual overlap in the caudal diencephalon (pretectum and $\mathrm{pTh}-\mathrm{C}$ ). 

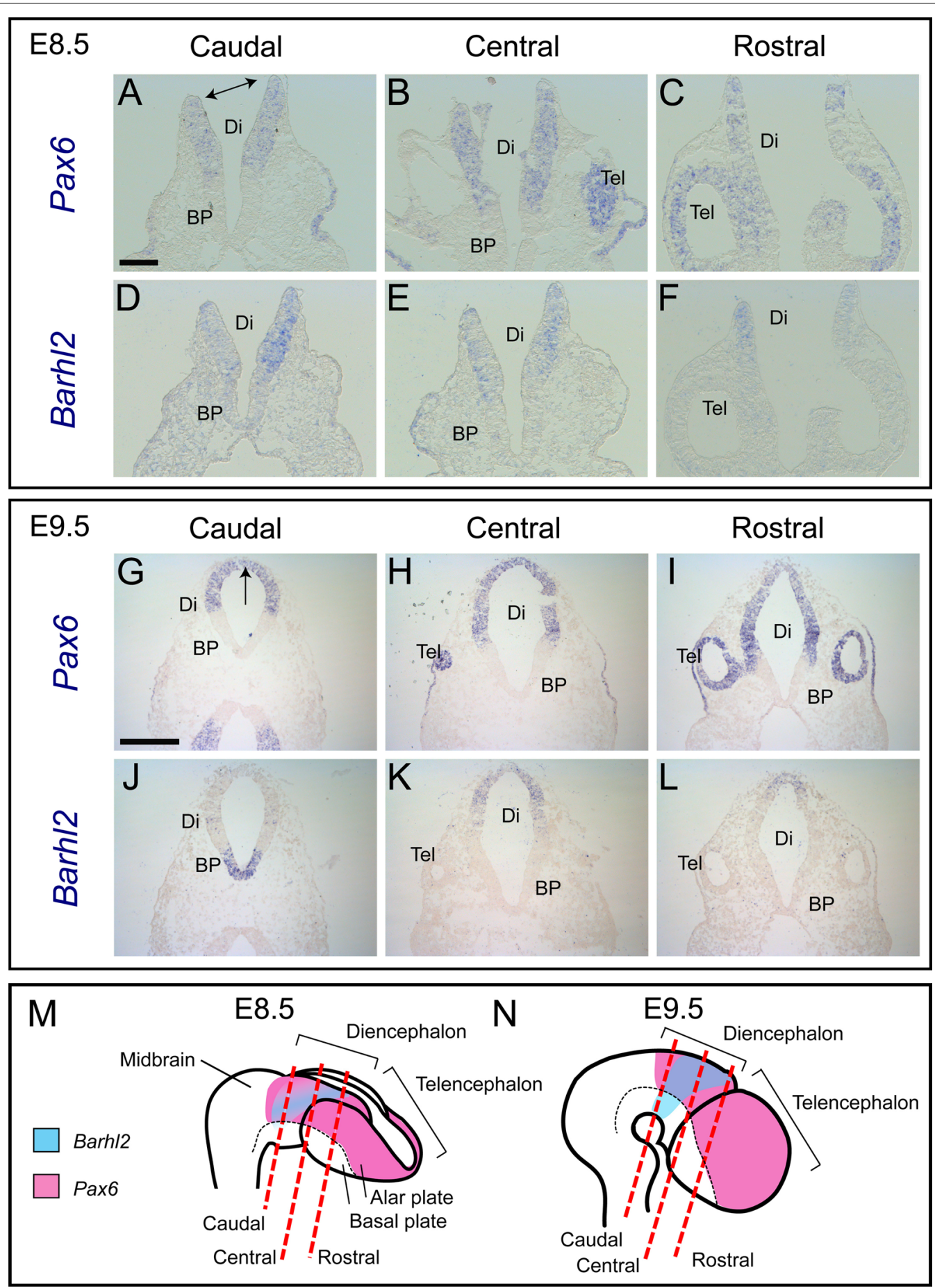

Fig. 1 A-L In situ hybridization data for Pax6 and Barh/2 mRNA in adjacent sections cut from embryos at E8.5 and E9.5. M, N Schematics to illustrate the planes of the sections in $\mathbf{A}-\mathbf{L}$ and to summarize the results. Scale bar for $\mathbf{A}-\mathbf{F} 200 \mu \mathrm{m}, \mathbf{G}-\mathbf{L} 500 \mu \mathrm{m}$. Tel telencephalon, Di diencephalon, BP basal plate

By E11.5 (Fig. 2G-L), the complementarity of Pax6 and Barhl2 expression has become increasingly obvious throughout much of the forebrain. Whereas many telencephalic progenitor cells express Pax6, they do not express Barhl2. Barhl2 is, however, now expressed by differentiating cells in the mantle zone of the ventral 

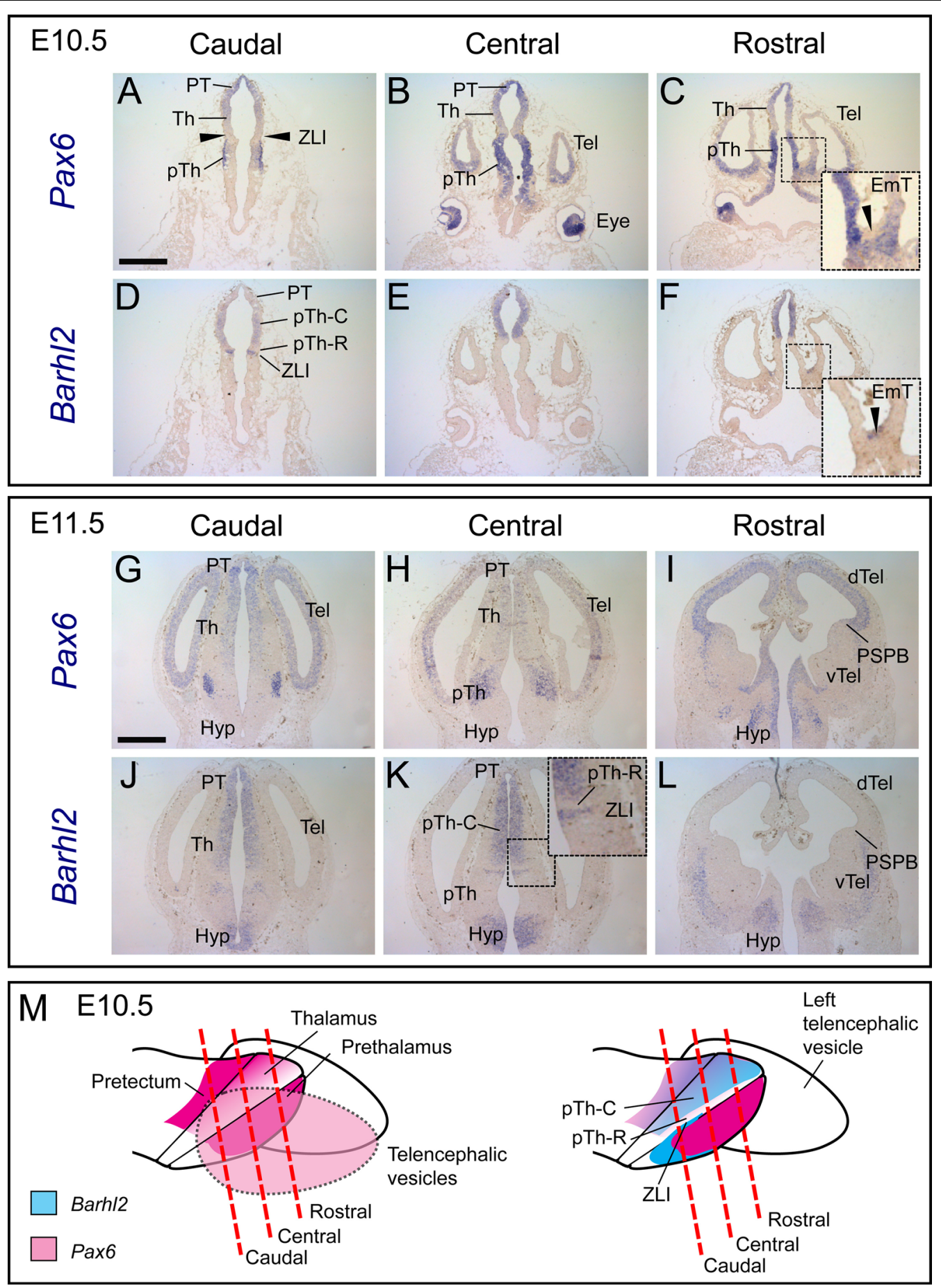

Fig. 2 A-L In situ hybridization data for Pax6 and Barh/2 mRNA in adjacent sections cut from embryos at E10.5 and E11.5. M Schematics to illustrate the planes of the sections and to summarize results at E10.5. Scale bars $500 \mu \mathrm{m}$. PT pretectum, Th thalamus, pTh prethalamus, ZLI zona limitans intrathalamica, EmT eminentia thalami, Hyp hypothalamus, PSPB pallial-subpallial boundary, Tel telencephalon, vTel ventral telencephalon, dTel dorsal telencephalon

telencephalon, in regions where Pax6 is not expressed (Fig. 2I, L). Pax6 and Barhl2 expression patterns in the hypothalamus also show striking complementarity
(Fig. 2G-lL. In the diencephalon, Pax6 continues to be strongly expressed within the prethalamus, where Barhl2 is not expressed (Fig. 2H, K). The exception is the 
pretectum and the $\mathrm{pTh}-\mathrm{C}$ domain of the thalamus, where both genes are expressed (Fig. 2G, H, J, K). Barhl2 and Pax6 both remain low or absent from the pTh-R (Fig. 2H, $\mathrm{K})$ but only Barhl2 is strongly expressed within the ZLI (Fig. 2K).

The complementarity of Pax6 and Barhl2 expression patterns in the telencephalon, eminentia thalami, hypothalamus and prethalamus is well-developed at E12.5E13.5 (Fig. 3). Neither gene is expressed specifically in progenitor or postmitotic zones: for example, $\operatorname{Pax} 6$ is expressed in both zones in the prethalamus (Fig. 3A-C, $\mathrm{G}, \mathrm{H}$ ) but in progenitor zones alone within the dorsal telencephalon and thalamus (Fig. 3B). Barhl2 is expressed in the progenitor zone of the thalamus (Fig. 3E) but in the postmitotic zone of the eminentia thalami (Fig. 3F, L). Overlap between Pax6 and Barhl2 expression patterns continues in the pretectum and pTh-C: Pax6 is still expressed in a gradient but its levels are relatively low compared to those in other forebrain regions (Fig. 3B) whereas levels of Barhl2 are as high or higher than those in other regions such as the ZLI (Fig. 3E, J) and eminentia thalami (Fig. 3F, L). Barhl2 expression levels appear to be graded across pTh-C by E12.5, with levels increasing from caudal to rostral sections (Fig. 3D-F).

\section{Co-expression of Pax6 and Barh/2 in the diencephalon}

The analysis above indicates that diencephalic patterns of expression and co-expression of Pax6 and Barhl2 are complex and dynamic. To confirm and clarify the conclusions drawn from single-colour in situ hybridizations on adjacent coronal sections, we carried out fluorescence double-labelling with both probes on parasagittal sections of the brains of embryos of increasing age (Fig. 4).

At E9.5, this analysis confirmed conclusions from coronal sections (summarised in Fig. 1N). Pax6 is expressed throughout alar diencephalon and Barhl2 is expressed in a narrower region, extending from the floorplate to the roofplate, whose alar domain overlaps a central portion of the diencephalic domain of Pax6 expression (Fig. 4AC). At high magnification expression of both Pax6 and Barhl2 could be seen in the presumptive ZLI (outlined area, Fig. 5A) and also in the region of prethalamic neuroepithelium directly rostral to it (Fig. 5B-D). Barhl2 mRNA expression is detected primarily in the cytoplasm whereas Pax6 is located in the nucleus (Fig. 5B, D). In the alar prethalamic neuroepithelium, all cells expressed Pax6 at this age (Fig. 5C, D). Given the fact that Barhl2 is present in surrounding cytoplasm between the Pax6-positive nuclei, we can deduce that many cells in this region co-express Pax6 and Barhl2 (Fig. 5C, D).

At E10.5 the ZLI emerges as a thin Barhl2-positive, Pax6-negative domain between the Barhl2-positive thalamus and the prethalamus, which is now Barhl2-negative
(Fig. 4D-F). This confirms conclusions summarized in Fig. 2M. The separation of the Barhl2-positive ZLI from the Barhl2-positive pTh-C by the pTh-R, a narrow strip of tissue expressing low or no Pax6 and Barhl2, becomes clearer by E11.5 (Fig. 4G-I). The Barhl2-positive ZLI continues to be obvious at E12.5-13.5 (Fig. 4J-O).

We next considered the relationship between the expression of Pax6 and Barhl2 in the pretectum and thalamus where, unusually, both are expressed in the same region for a prolonged period. We first considered whether Pax6 and Barhl2 are expressed by the same cells in the pretectum and thalamus by double-labelling for Pax6 protein and Barhl2 mRNA in the same coronal sections at E12.5 (Fig. 6). In these experiments we did not delineate the exact position of the boundary between the pretectum and thalamus, preferring to analyse the two together since the gradients of Pax6 and Barhl2 expression were continuous across the two regions (Fig. 6A). As shown in Fig. 6A, C, G, cells express higher levels of Pax6 the closer they are to the pretectum. Close to the pretectum, almost all cells express detectable levels of Pax6 (Fig. 6B, C). These cells also stain for Barhl2 in the cytoplasm around their Pax6-positive nuclei (Fig. 6D, E). This contrasts with other forebrain regions, such as the eminentia thalami, where Pax6-expressing and Barhl2expressing cells are clearly segregated (Fig. 6J-M).

To study the relationship between the gradient of Pax6 and expression levels of Barhl2 across the thalamus, we examined coronal sections double-labelled with immunohistochemistry for Pax6 and fluorescence in situ hybridization for Barhl2 (Fig. 7). The plane at which these sections were cut (Fig. 7M) offers a clear view of the gradient of Pax6 expression (Fig. 7A, D, G, J) and, therefore, the opportunity to correlate this gradient with variations in Barhl2 expression. In the examples shown in Fig. 7 (and also that shown in Fig. 6) there is evidence of countergradients of Barhl2, with levels increasing towards the ZLI, at E10.5-E12.5. By E13.5, Pax6 levels in the thalamus are very low and there is no longer any obvious gradient of Barhl2, which is relatively strongly expressed in the ventricular zone of the thalamus (Fig. 7J-L).

To gain more objective data on these countergradients and their variation between embryos of the same and different ages, we quantified the levels of Pax6 and Barhl2 expression in three embryos at each of four ages, E10.5, E11.5, E12.5 and E13.5 (Fig. 8).

At all the ages studied-even at E13.5, when Pax6 levels are overall low throughout the thalamus (Fig. 7J) the intensity of staining for Pax6 showed a consistently negative correlation with distance from pretectum to ZLI (Fig. 8B-D). This is shown by the green lines and the mean gradients for each individual embryo (Fig. 8B, C) and by relatively low variance around the means at each 

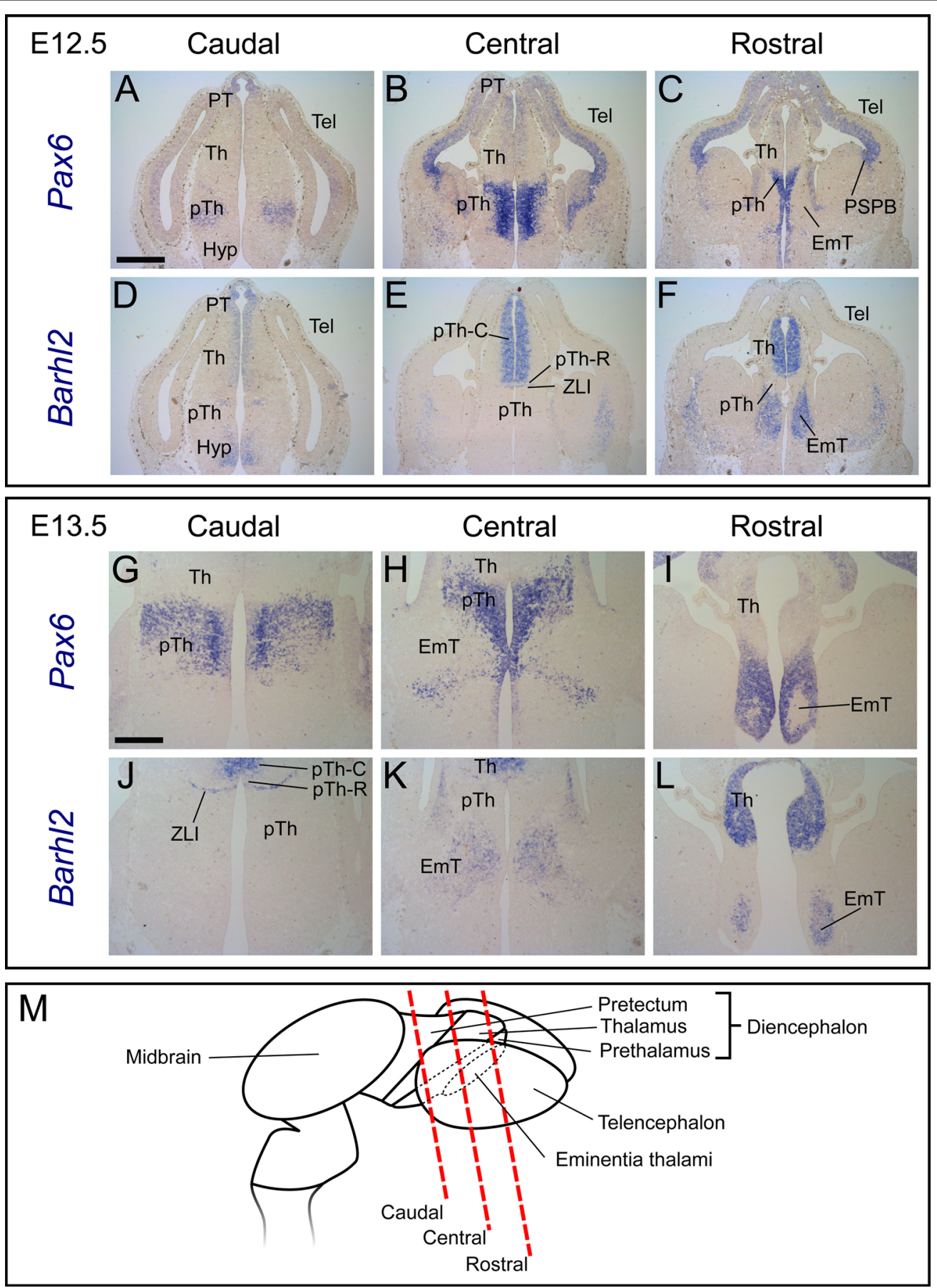

Fig. 3 A-L In situ hybridization data for Pax6 and Barh/2 mRNA in adjacent sections cut from embryos at E12.5 and E13.5. M Schematic to illustrate the planes of the sections. Scale bar for A-F $500 \mu \mathrm{m}, \mathbf{G}-\mathbf{L} 250 \mu \mathrm{m}$. PT pretectum, Th thalamus, pTh prethalamus, Hyp hypothalamus, ZLI zona limitans intrathalamica, EmT eminentia thalami, PSPB pallial-subpallial boundary, Tel telencephalon

(See figure on next page.)

Fig. 4 A-O Sagittal sections of embryos treated with immunostaining for Pax6 protein and in situ hybridization for Barh/2 mRNA. P Schematic to illustrate the approximate plane of section. Scale bars for A-I $250 \mu \mathrm{m}, \mathbf{J}-\mathbf{0} 500 \mu \mathrm{m}$. Tel telencephalon, Di diencephalon, Mes mesencephalon, BP basal plate, AP alar plate, PT pretectum, Th thalamus, pTh prethalamus, ZLI zona limitans intrathalamica 


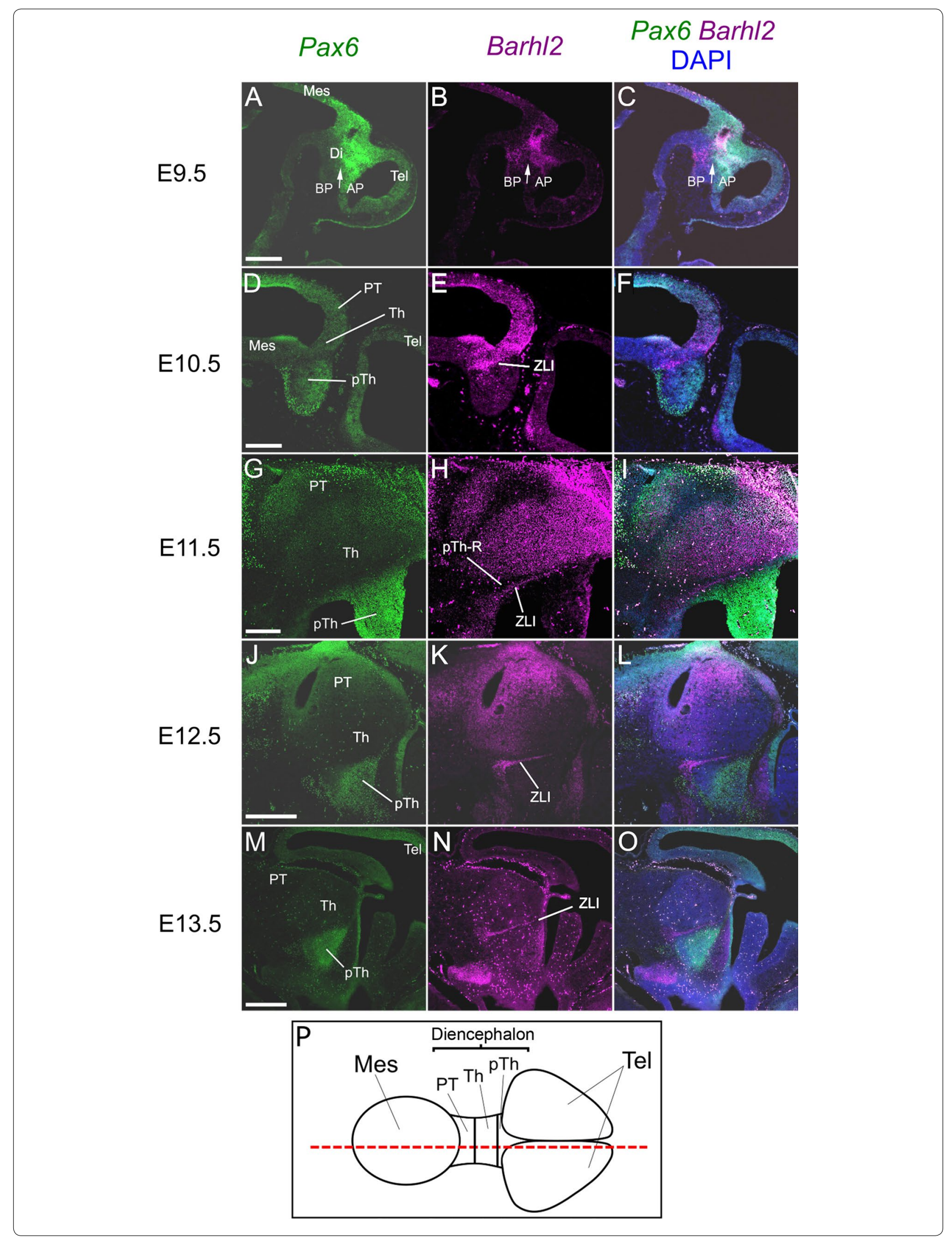




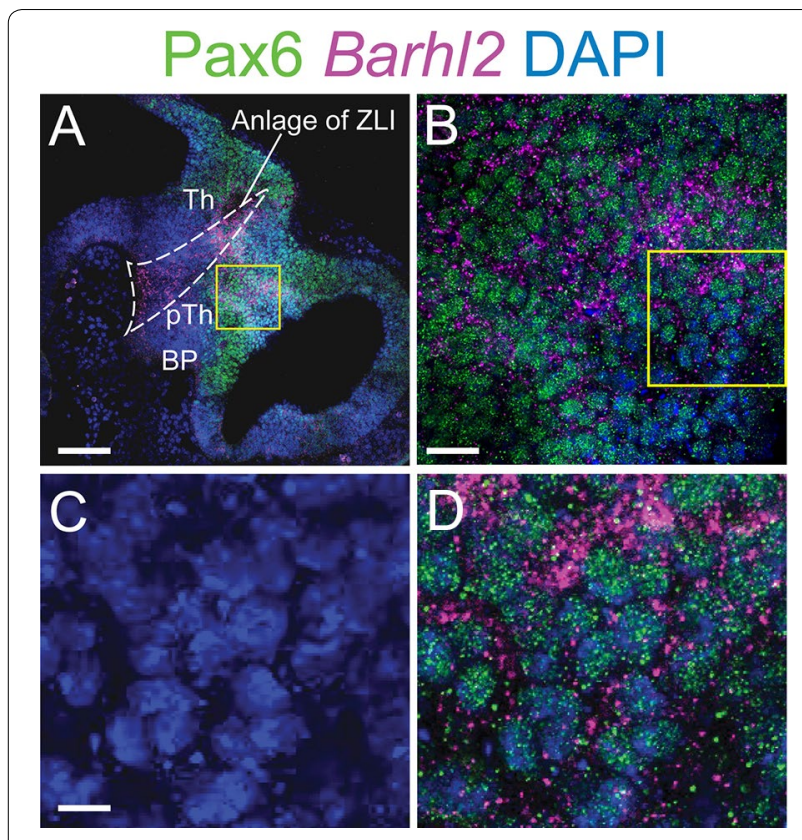

Fig. 5 A Sagittal section of E9.5 embryo immunostained for Pax6 protein and in situ hybridization for Barh/2 mRNA. Scale bar $200 \mu \mathrm{m}$. B Detail of area outlined in A. Scale bar $25 \mu \mathrm{m}$. C, D DAPI staining and triple-staining for DAPI, Pax6 and Barh/2 within the prethalamus in the area outlined in B. Scale bar $10 \mu \mathrm{m}$ age (green bars in Fig. 8D). At E12.5, all three embryos show a countergradient of Barhl2 (Fig. 8B-D), as shown by the magenta lines and the mean gradients for each E12.5 embryo in Fig. 8B, C and by the low variance around the mean at E12.5 (third magenta bar in Fig. 8D). At earlier ages, clear gradients of Barhl2 expression were not always detected, although where strong gradients were detected they ran counter to those of Pax6 (Fig. 8B, C). At the latest age examined, E13.5, when Pax6 levels are generally very low (Fig. 7J), no countergradients were observed (Fig. 8B-D). These data suggest that an inverse relationship between thalamic gradients of Pax6 and Barhl 2 becomes established over the 2 days between E10.5 and E12.5, with variability in the timing of its emergence between individuals. The gradient of Pax6 appears to be established robustly before that of Barhl2.

\section{Expression of Barh/2 in the Pax6-null forebrain}

The findings above suggested that Pax6 might repress the forebrain expression of Barhl2. In order to investigate this possibility further we performed in situ hybridization for Barhl2 mRNA on cryosections from $\mathrm{Pax}^{\mathrm{Sey} / \mathrm{Sey}}$ forebrains (Fig. 9).

As has been described before, the morphology of the $P a x 6^{S e y / S e y}$ mutant forebrain differs from that of the

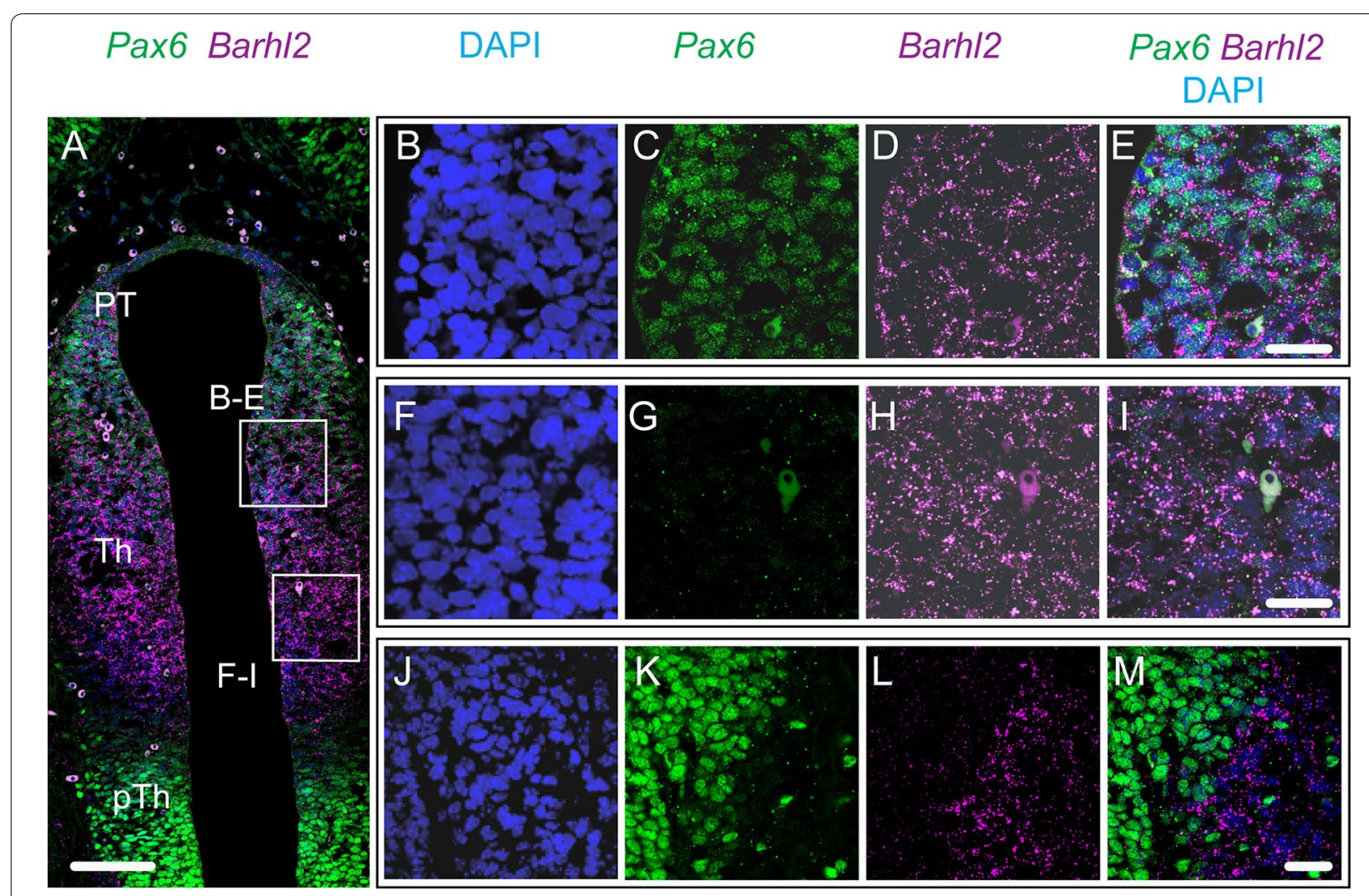

Fig. 6 A Coronal section of E12.5 embryo treated with immunostaining for Pax6 protein and in situ hybridization for Barh/2 mRNA. B-E, F-I Higher magnification of tissue outlined in A. J-M Detail of the eminentia thalami. Scale bars for A $100 \mu \mathrm{m}, \mathbf{B}-\mathbf{M} 25 \mu \mathrm{m}$. PT pretectum, Th thalamus, pTh prethalamus 


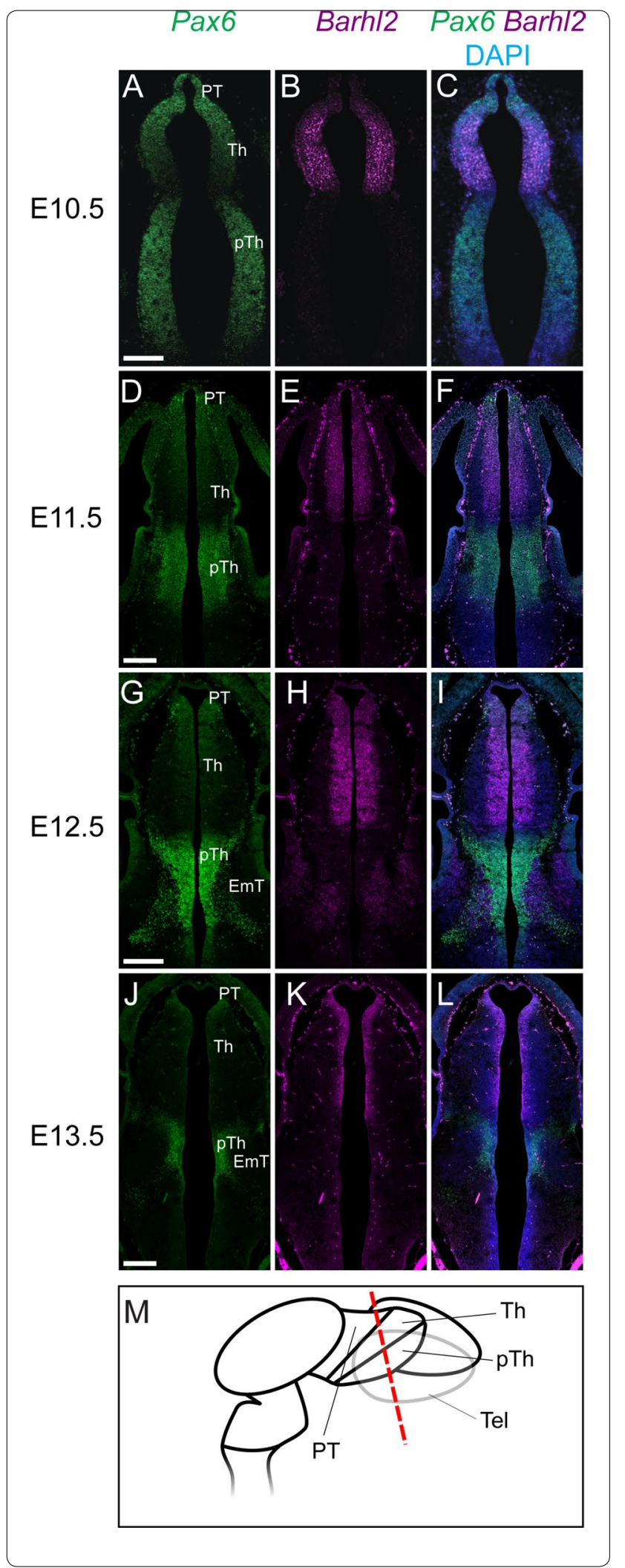

Fig. 7 A-L Coronal sections of embryos treated with immunostaining for Pax6 protein and in situ hybridization for Barh/2 mRNA. M Schematic to illustrate the approximate plane of section. Scale bars $500 \mu \mathrm{m}$. PT pretectum, Th thalamus, pTh prethalamus, ZLI zona limitans intrathalamica, EmT eminentia thalami, Tel telencephalon

wild-type. The ZLI is expanded [22-24], much of the neuroepithelium is reduced in thickness $[41,42]$ and the third ventricle expands laterally [43] as a result of two diencephalic structures, the paraventricular nucleus and the caudal zona incerta, failing to develop correctly [44]. Despite these differences in morphology, structures such as the thalamus and prethalamus can be distinguished in both the wild type and mutant forebrain [22, 44], making it possible to compare the expression of Barhl2 in these regions. Nestin staining for radial glial cells in the ventricular zone [45] was similar in $\mathrm{Pax6}^{+/+}$and $\mathrm{Pax}_{6}^{\mathrm{Sey} / \mathrm{Sey}}$ embryos and indicated that there was no major change in the depth of the ventricular zone in mutants (Fig. 10).

In the E11.5-E12.5 $\mathrm{Pax}^{+/+}$diencephalon the domain of Barhl2 within the pTh-C was restricted to the ventricular zone (Fig. 9A, B, G, H). In the Pax6 ${ }^{\text {Sey/Sey }}$ mutant the thalamic Barhl2 domain spanned all or most of the mediolateral width of the thalamic neuroepithelium, most likely due to the absence of normal mantle zone development (Fig. 9D, E, J, K). Barhl2 was expressed within the expanded ZLI of $\mathrm{Pax} 6^{\mathrm{Sey} / \mathrm{Sey}}$ mutants. The most striking difference between Barhl2 expression in $\mathrm{Pax}^{+/+}$and $\mathrm{Pax} 6^{\mathrm{Sey} / \mathrm{Sey}}$ diencephalon was its elevated expression in the thalamus and pretectum of mutants at E12.5, but not earlier. The pTh-R was clearer in the $\mathrm{Pax6}^{+/+}$diencephalon, where it was Barhl2-negative, than in the Pax $6^{\mathrm{Sey} / \mathrm{Sey}}$ diencephalon, where it showed weaker Barhl2 expression than surrounding pTH-C and ZLI (Fig. 9B, E, H, K). The pattern of Barhl2 expression in other forebrain regions appeared similar in both genotypes at E11.5-E12.5 (allowing for the morphological differences). The prethalamus, dorsal telencephalon and much of the ventral telencephalon (with the exception of ventro-laterally positioned cells which expressed Barhl2 in both genotypes; Fig. 9C, F, I, L) remained negative for Barhl2 in the mutants. We conclude that the loss of Pax6 causes increased Barhl2 specifically in the pretectum and thalamus, where their co-expression is normally prolonged.

\section{Comparison of Barhl2 and Ngn2 expression in the embryonic forebrain}

Barhl2 has been suggested as an inhibitor of the expression of bHLH transcription factors. We compared its diencephalic expression to that of the bHLH transcription factor Neurogenin2 (Ngn2) using double fluorescence 


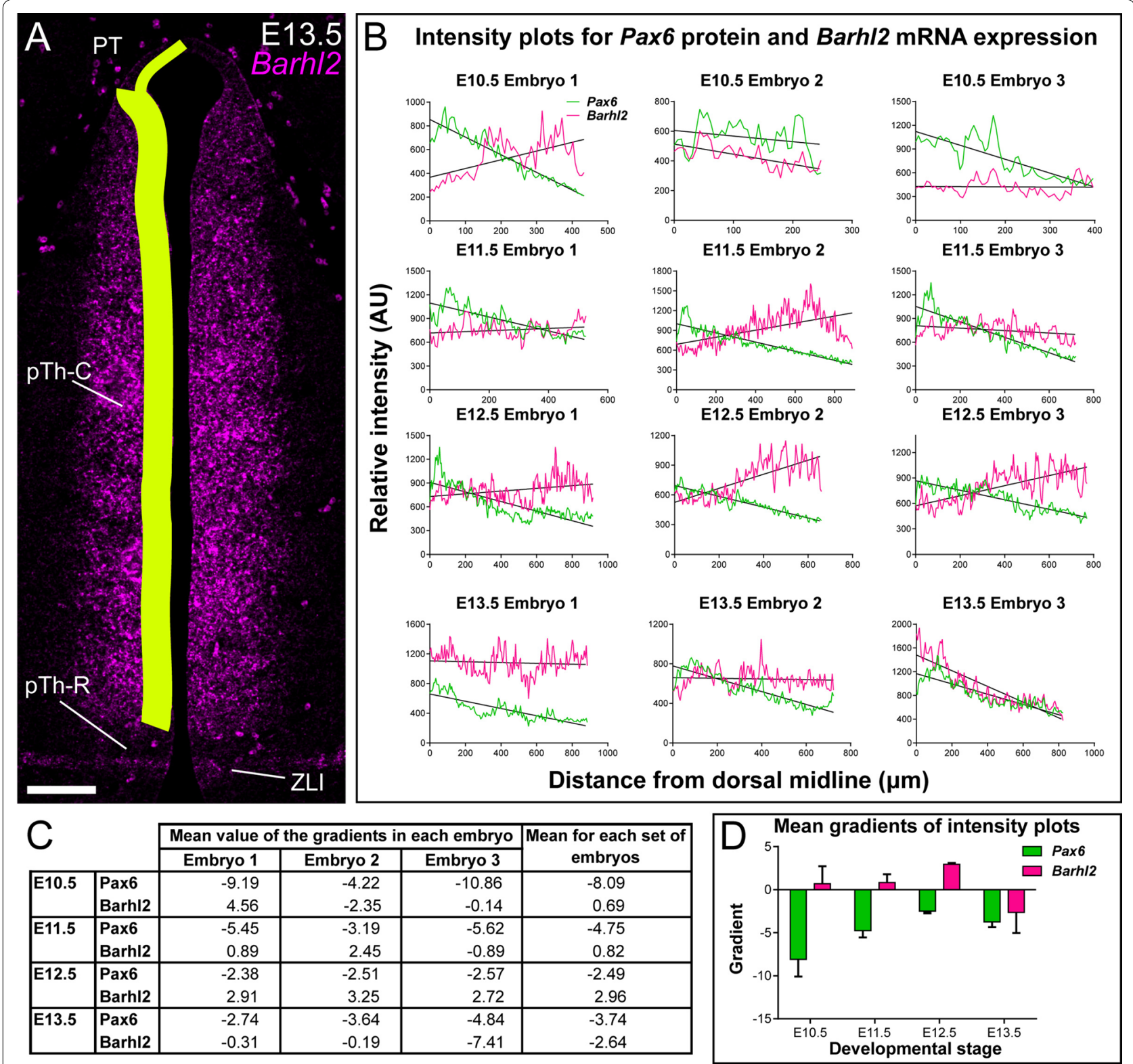

Fig. 8 A Schematic to illustrate the area of the ventricular zone across which the intensity plots were calculated. Scale bar $100 \mu \mathrm{m}$. B Intensity plots for 12 embryos, three at each of four ages from E10.5 to E13.5, with linear regression trend lines. CTable of the gradients of the trend lines for each developmental stage, and the mean gradient for each set of three embryos. D Bar chart of the mean values of the gradients recorded for each group of three embryos, with standard errors. PT pretectum, ZLI zona limitans intrathalamica, dpc days post conception

in situ hybridization with riboprobes for Barhl2 and $\mathrm{Ngn} 2$ on sections cut at the level of the central diencephalon from embryos at E11.5, E12.5 and E13.5 (Fig. 11). In the telencephalon and the eminentia thalami, expression of Barhl2 complemented that of Ngn2 (Fig. 11C, F, I). $\mathrm{Ngn} 2$ was expressed in the dorsal telencephalon and the proliferative zone of the eminentia thalami. Barhl2, on the other hand, was not expressed in dorsal telencephalon and was expressed in the differentiating but not the proliferating zone of the eminentia thalami.
In the diencephalon, however, the expression patterns of Barhl2 and Ngn2 were similar. Neither Barhl2 nor Ngn2 was expressed in the prethalamus and the pTh- $R$, while both were expressed in the ZLI and pTh-C (Fig. 11A-L). Within the ZLI, Barhl2 expression corresponded with that of $\mathrm{Ngn2}$, which is known to be expressed mainly in the more caudal region of the ZLI [22]. To confirm the location of Barhl2 expression in the ZLI, double fluorescence in situ hybridization was performed using riboprobes for Barhl2 and 

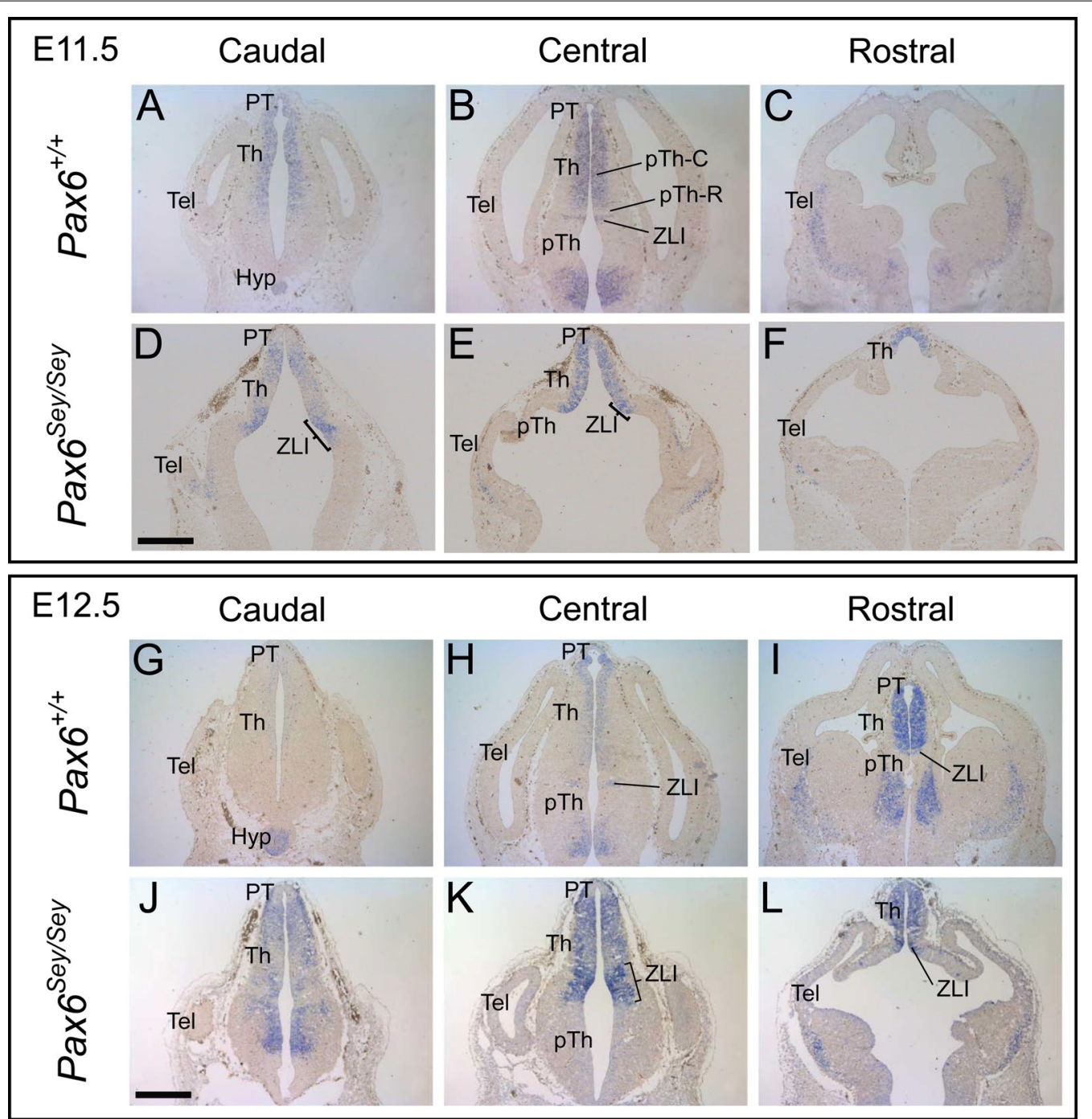

Fig. 9 A-F In situ hybridization for Barh/2 mRNA on comparable sections from Pax $6^{+/+}$and Pax ${ }^{\text {Sey/Sey }}$ embryos at E11.5. G-L In situ hybridization for Barh/2 mRNA on comparable sections from Pax6 $6^{+/+}$and Pax6 $6^{\text {Sey/Sey }}$ embryos at E12.5. PT pretectum, Th thalamus, pTh prethalamus, ZLI zona limitans intrathalamica, EmT eminentia thalami, Tel telencephalon, Hyp hypothalamus. Scale bars $500 \mu \mathrm{m}$

Shh, the definitive marker of the entire ZLI [21, 22]. The domain of Barhl2 was slightly narrower than that of Shh within the ZLI and was centred caudally, as anticipated (Fig. 11M-O). In the thalamus and pretectum, Barhl2 expression was confined to the proliferative zone whereas $\mathrm{Ngn} 2$ expression extended through the proliferative zone and into the overlying differentiating mantle zone.

\section{Discussion}

\section{Evidence for interactions between Barhl2 and Pax6}

The expression of Barhl2 complements that of Pax6 in the telencephalon and eminentia thalami from early stages of its development, but the expression domains of Barhl2 and Pax6 overlap in diencephalic regions. Early in development, there is co-expression of the two genes from prethalamus to pretectum, but Barhl2 expression is rapidly turned off in prethalamus, where Pax6 levels remain high. Co-expression persists in the thalamus and pretectum, where Pax6 and Barhl2 develop countergradients. Our data suggest that a robust gradient of Pax6 expression across this region predates the establishment of a robust countergradient of Barhl2 expression. Soon after consistent countergradients are established, Pax6 levels become undetectable across most of this region, while Barhl2 levels remain high. These results suggested that Pax6 expression might have a repressive effect on Barhl2 expression. This might be direct or indirect. A screen designed to predict Pax6 target genes identified 


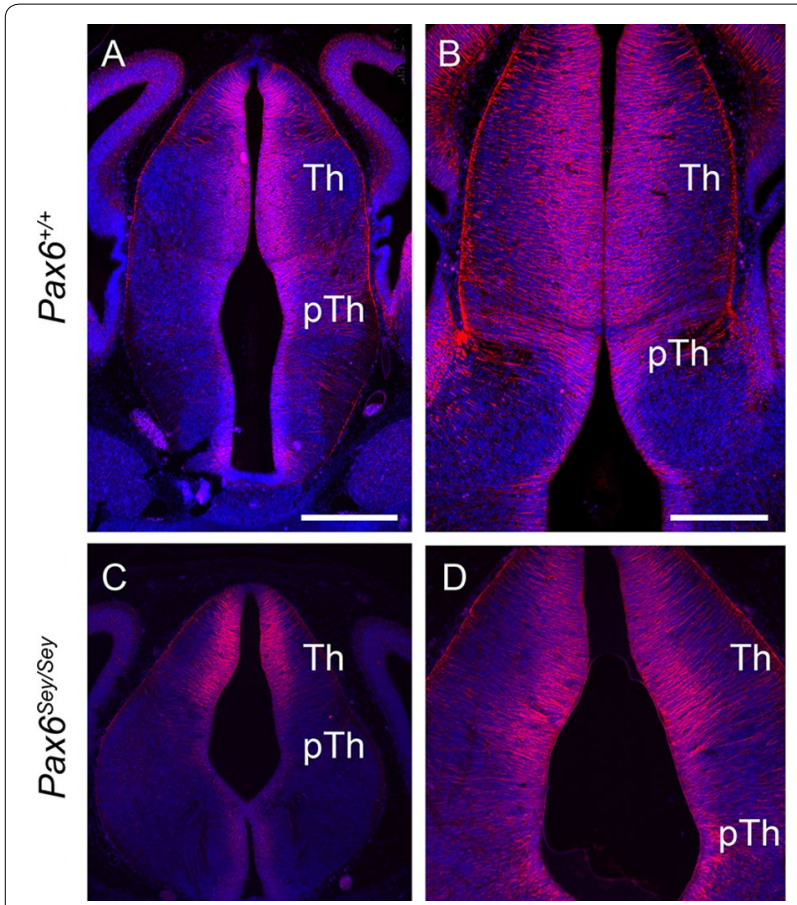

Fig. 10 Immunohistochemistry for Nestin on sections of the E13.5 $\mathrm{Pax6}^{+/+}$and Pax6 ${ }^{\text {Sey/Sey }}$ diencephalon. Th thalamus, PTH prethalamus. Scale bars $500 \mu \mathrm{m}$ in $\mathbf{A}, \mathbf{C}$ and $250 \mu \mathrm{m}$ in B, D

13 potential Pax6 binding sites around the transcription start site of Barhl2, suggesting that Pax6 protein may be capable of exerting a direct effect on the expression of Barhl2 mRNA [46].

We found that the absence of Pax6 did not induce Barhl2 expression in telencephalic regions that normally express Pax6 but not Barhl2. Nor did the absence of Pax 6 prevent the loss of Barhl2 expression from the prethalamus. Absence of Pax6 did, however, increase Barhl2 expression in the thalamus and pretectum. This interesting finding suggests a functional interaction between Pax6 and Barhl2 only in the region of the forebrain in which co-expression of the two genes persists much longer than in any other region. Whereas factors other than Pax6 appear to control the overall expression patterns of Barhl2, Pax6 is likely to play an important part in modulating the expression levels of Barhl2 in just this one specific part of the forebrain. Our findings indicated that absence of Pax6 led to Barhl2 upregulation in thalamus and pretectum after E11.5, suggesting that Pax6 exerts its repressive effect on Barhl2 only once the production of neurons is getting underway. The importance of this might be to limit the anti-proneural activity of Barhl2, reducing its ability to interfere with the proneural activity of factors such as Ngn2. It is possible that proneural inhibition by unfettered upregulation of Barhl2 expression in the absence of Pax6 is a cause of the reduced size of the differentiating (mantle) zone in Pax6-null mutants.

Previous studies have provided limited evidence for the possibility that Barhl2 and Pax6 might be mutually repressive. One study reported an expansion of the Pax6 expression domain in response to a morpholino knockdown of Xbarhl2 in the Xenopus embryo [47]. Further work is needed to test whether loss of Barhl 2 in mice affects the expression of Pax6.

The relationship between Barhl2 and Pax6 expression in the pretectum and thalamus may differ from that observed in other forebrain regions for many reasons. The regions of neuroepithelium fated to become the thalamus and prethalamus are established prior to ZLI formation and differ significantly in molecular character from the forebrain regions rostral to the ZLI [48]. The thalamic anlage expresses both $\operatorname{Ir} x 3$ and Pax6, which together confer competence to develop into the thalamus in response to the Shh signal [37]. The prethalamic anlage strongly expresses Pax6 but not Irx3 [36] and also expresses Six3, which confers competence on the neuroepithelium to develop into more rostral forebrain structures [42]. Both regions of neuroepithelium are competent to respond to secreted signals from the ZLI [21], but respond in different ways, and signalling from the ZLI therefore exerts changes in gene expression in an asymmetric fashion [39]. These differences in molecular character may also influence the degree to which Pax6 and Barhl2 can be co-expressed.

\section{Barh/2 and Pax6 in ZLI development}

The ZLI is a thin Shh-expressing strip of tissue that separates the thalamus and prethalamus. Morpholino knockdown of Barhl2 expression in Xenopus results in a failure of the ZLI to develop [20], while the loss of functional Pax6 in mice causes the ZLI to undergo an expansion along the rostrocaudal axis of the diencephalon [22-24]. Together these findings suggest that Barhl2 is required for ZLI initiation, while Pax6 may play a later role in the shaping of the ZLI. It is possible that Pax6's inhibition of Barhl2 might contribute to its role in limiting the region of diencephalon that is competent to develop into the ZLI. In the developing Drosophila retina $\mathrm{Hh}$ acts upstream of $\mathrm{BarH} 1$ and $\mathrm{BarH} 2$ and is required to initiate their expression [49]. In the vertebrate forebrain the need for Barhl2 in the induction of the ZLI suggests that Barhl2 may act upstream of Shh in this process and may be required to induce 


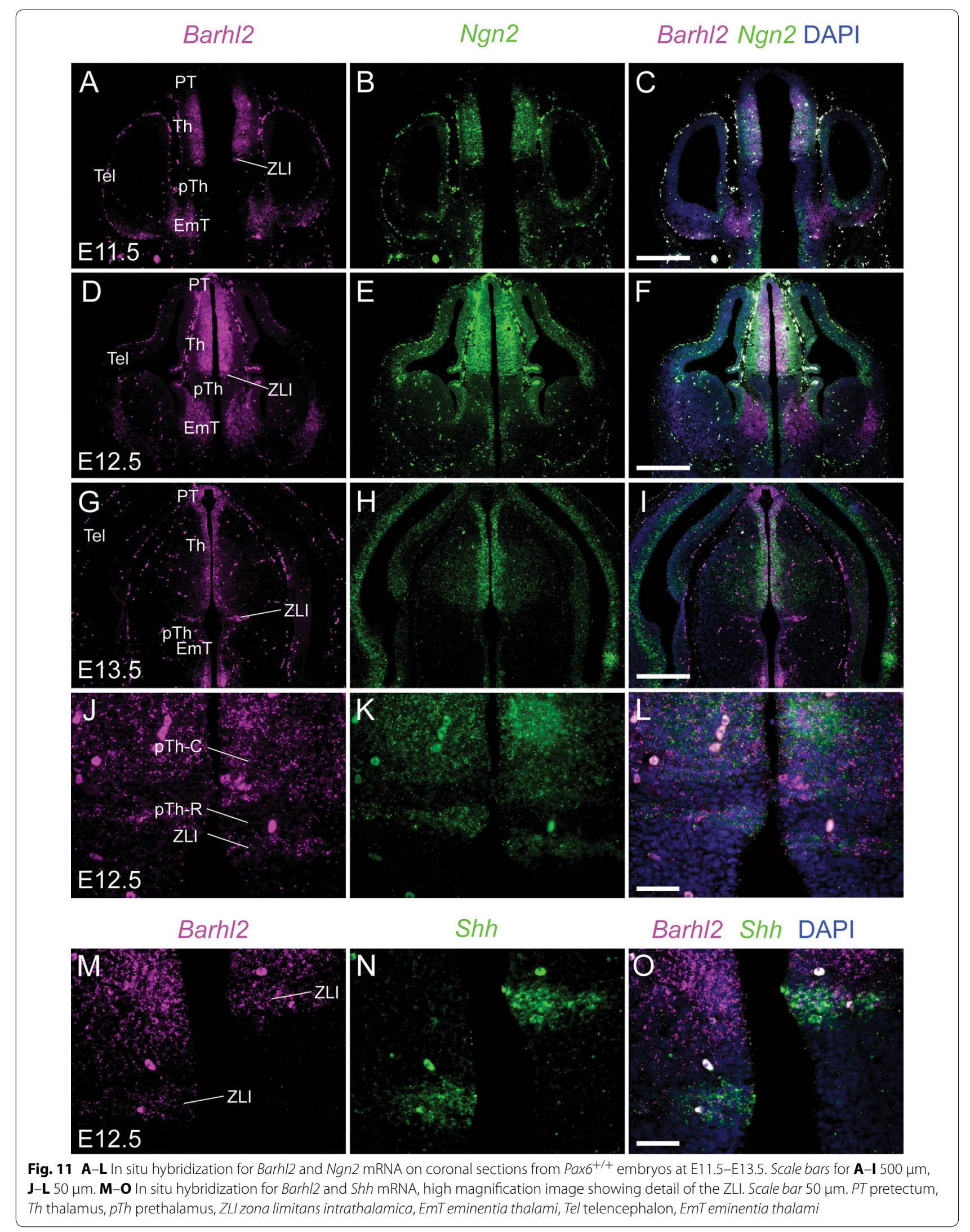


Shh expression. Alternatively, Barhl2 may be required to render a central region of diencephalic neuroepithelium competent to develop into ZLI.

While Barhl2 is expressed in the anlage of the ZLI, and later within the mature ZLI itself, it is not expressed throughout the whole of the mature ZLI. Its expression is confined to the more caudal, Ngn2-expressing region. It is not clear what function Barhl2 serves in the mature ZLI, if any, and why it is not expressed throughout the entire ZLI.

\section{Conclusions}

The expression of Barhl2 in the thalamus and pretectum is related to, and regulated by, the expression of Pax6. Pax6 is known to be required for normal diencephalic development. The findings presented here suggest that some of its actions might be mediated by its maintenance of a repressive influence over Barhl2 expression in the thalamus and pretectum.

\begin{abstract}
Abbreviations
bHLH: basic helix-loop-helix; AC: amacrine cell; PBS: phosphate-buffered saline; OCT: optimal cutting temperature; Tel: telencephalon; Di: diencephalon; Mes: mesencephalon; Rh: rhombencephalon; SC: spinal cord; PT: pretectum; Th: thalamus; pTh: prethalamus; ZLI: zona limitans intrathalamica; EmT: eminentia thalami; Hyp: hypothalamus; vTel: ventral telencephalon; dTel: dorsal telencephalon; PSPB: pallial-subpallial boundary; BP: basal plate; AP: alar plate; dpc: days post conception.

\section{Authors' contributions}

EVP designed and carried out all experiments, recorded all image data and performed the quantitative analysis. DJP and JOM advised on the design of experiments. DJP directed the study. EVP and DJP drew the illustrations and compiled the figures. All authors read and approved the final manuscript.
\end{abstract}

\section{Acknowledgements}

We thank Trudi Gillespie and Anisha Kubasik-Thayil of the Image Analysis Multi Photon and Confocal Technologies (IMPACT) facility at The University of Edinburgh for their assistance in obtaining the confocal image data. We thank Asuka Suzuki-Hirano and Tomomi Shimogori of the RIKEN Brain Science Institute, Wako-shi, Saitama, Japan for providing us with the Barh/2 riboprobe and for their advice on the optimisation of the Barh/2 in situ hybridization protocol.

\section{Competing interests}

The authors declare that they have no competing interests.

\section{Availability of data and materials}

Raw counts and images are stored on the University of Edinburgh filestore and will be made freely available on request to DJP.

\section{Ethics approval}

All protocols were reviewed and approved by the University Ethics Committee, which includes the named veterinary surgeons of the College of Medicine and Veterinary Medicine, the University of Edinburgh, and by the UK Home Office prior to the commencement of experimental work.

\section{Funding}

The work presented here was funded by a Medical Research Council Capacity Building Studentship to EVP and Medical Research Council Grants J003662 and NO12291 to DJP and JOM.
Received: 2 August 2016 Accepted: 16 November 2016

Published online: 25 November 2016

\section{References}

1. Briscoe J, Small S. Morphogen rules: design principles of gradient-mediated embryo patterning. Development. 2015;142:3996-4009.

2. Rebeiz M, Patel NH, Hinman VF. Unraveling the tangled skein: the evolution of transcriptional regulatory networks in development. Annu Rev Genomics Hum Genet. 2015;16:103-31.

3. Schuhmacher L-N, Albadri S, Ramialison M, Poggi L. Evolutionary relationships and diversification of barhl genes within retinal cell lineages. BMC Evol Biol. 2011;11:340

4. Suzuki-Hirano A, Ogawa M, Kataoka A, Yoshida AC, Itoh D, Ueno M, Blackshaw S, Shimogori T. Dynamic spatiotemporal gene expression in embryonic mouse thalamus. J Comp Neurol. 2011;519:528-43.

5. Reig G, Cabrejos ME, Concha ML. Functions of BarH transcription factors during embryonic development. Dev Biol. 2007;302:367-75.

6. Smith ST, Jaynes JB. A conserved region of engrailed, shared among all en-, gsc-, Nk1-, Nk2- and msh-class homeoproteins, mediates active transcriptional repression in vivo. Development. 1996;122:3141-50.

7. Kobayashi M, Nishikawa K, Suzuki T, Yamamoto M. The homeobox protein Six3 interacts with the groucho corepressor and acts as a transcriptional repressor in eye and forebrain formation. Dev Biol. 2001;232:315-26.

8. Muhr J, Andersson E, Persson M, Jessell TM, Ericson J. Groucho-mediated transcriptional repression establishes progenitor cell pattern and neuronal fate in the ventral neural tube. Cell. 2001;104:861-73.

9. Bae Y-K, Shimizu T, Yabe T, Kim C-H, Hirata T, Nojima H, Muraoka O, Hirano T, Hibi M. A homeobox gene, pnx, is involved in the formation of posterior neurons in zebrafish. Development. 2003;130:1853-65.

10. Higashijima S, Kojima T, Michiue T, Ishimaru S, Emori Y, Saigo K. Dual Bar homeo box genes of Drosophila required in two photoreceptor cells, R1 and R6, and primary pigment cells for normal eye development. Genes Dev. 1992;6:50-60.

11. Chen G, Courey AJ. Groucho/TLE family proteins and transcriptional repression. Gene. 2000;249:1-16.

12. Courey AJ, Jia S. Transcriptional repression: the long and the short of it. Genes Dev. 2001;15:2786-96.

13. Agarwal M, Kumar P, Mathew SJ. The Groucho/Transducin-like enhancer of split protein family in animal development. IUBMB Life. 2015;67:472-81.

14. Lim J, Choi K-W. Bar homeodomain proteins are anti-proneural in the Drosophila eye: transcriptional repression of atonal by bar prevents ectopic retinal neurogenesis. Development. 2003;130:5965-74.

15. Sommer L, Ma Q, Anderson DJ. Neurogenins, a novel family of atonalrelated $\mathrm{bHLH}$ transcription factors, are putative mammalian neuronal determination genes that reveal progenitor cell heterogeneity in the developing CNS and PNS. Mol Cell Neurosci. 1996:8:221-41.

16. Saito T, Sawamoto K, Okano H, Anderson DJ, Mikoshiba K. Mammalian $\mathrm{BarH}$ homologue is a potential regulator of neural bHLH genes. Dev Biol. 1998;199:216-25.

17. Ding $Q$, Chen H, Xie X, Libby RT, Tian N, Gan L. BARHL2 differentially regulates the development of retinal amacrine and ganglion neurons. J Neurosci. 2009;29:3992-4003.

18. Jusuf PR, Albadri S, Paolini A, Currie PD, Argenton F, Higashijima S, Harris WA, Poggi L. Biasing amacrine subtypes in the Atoh7-lineage through expression of Barhl2. J Neurosci. 2012;32:13929-44.

19. Ding Q, Joshi PS, Xie Z, Xiang M, Gan L. BARHL2 transcription factor regulates the ipsilateral/contralateral subtype divergence in postmitotic dl1 neurons of the developing spinal cord. Proc Natl Acad Sci USA. 2012;109:1566-71.

20. Juraver-Geslin HA, Gómez-Skarmeta JL, Durand BC. The conserved barHlike homeobox-2 gene barhl2 acts downstream of orthodentricle- 2 and together with iroquois-3 in establishment of the caudal forebrain signaling center induced by Sonic hedgehog. Dev Biol. 2014;396:107-20.

21. Kiecker $C$, Lumsden $A$. Hedgehog signaling from the $Z L I$ regulates diencephalic regional identity. Nat Neurosci. 2004;7:1242-9. 
22. Caballero IM, Manuel MN, Molinek M, Quintana-Urzainqui I, Da M, Shimogori T, Price DJ. Cell autonomous repression of Shh by transcription factor Pax6 regulates diencephalic patterning by controlling the central diencephalic organizer. Cell Rep. 2014;8:1405-18.

23. Grindley JC, Hargett LK, Hill RE, Ross A, Hogan BLM. Disruption of PAX6 function in mice homozygous for the Pax6Sey-1Neu mutation produces abnormalities in the early development and regionalization of the diencephalon. Mech Dev. 1997;64:111-26.

24. Pratt T, Vitalis T, Warren N, Edgar JM, Mason JO, Price DJ. A role for Pax6 in the normal development of dorsal thalamus and its cortical connections. Development. 2000;127:5167-78.

25. Walther C, Gruss P. Pax-6, a murine paired box gene, is expressed in the developing CNS. Development. 1991;113:1435-49.

26. Kawakami A, Kimura-Kawakami M, Nomura T, Fujisawa H. Distributions of PAX6 and PAX7 proteins suggest their involvement in both early and late phases of chick brain development. Mech Dev. 1997;66:119-30.

27. Duan D, Fu Y, Paxinos G, Watson C. Spatiotemporal expression patterns of Pax6 in the brain of embryonic, newborn, and adult mice. Brain Struct Funct. 2013;218:353-72.

28. Home Office (UK). Animals (scientific procedures) act. 1986. http://www. legislation.gov.uk/ukpga/1986/14/contents. Accessed 22 Feb 2016.

29. Charles River Laboratories, Inc. CD- $1{ }^{\circledR}$ IGS mouse model information sheet. 2011. http://www.criver.com/files/pdfs/rms/cd1/rm_rm_d_cd1_ mouse.aspx. Accessed 20th Feb 2015.

30. Hill RE, Favor J, Hogan BL, Ton CC, Saunders GF, Hanson IM, Prosser J, Jordan T, Hastie ND, van Heyningen $V$. Mouse small eye results from mutations in a paired-like homeobox-containing gene. Nature. 1991;354:5225. doi:10.1038/354522a0.

31. Carney RSE, Cocas LA, Hirata T, Mansfield K, Corbin JG. Differential regulation of telencephalic pallial-subpallial boundary patterning by Pax6 and Gsh2. Cereb Cortex. 2009;9:745-59.

32. Adutwum-Ofosu KK, Magnani D, Theil T, Price DJ, Fotaki V. The molecular and cellular signatures of the mouse eminentia thalami support its role as a signalling centre in the developing forebrain. Brain Struct Funct. 2015. doi:10.1007/s00429-015-1127-3.

33. Pinson J, Mason JO, Simpson TI, Price DJ. Regulation of the Pax6:Pax6(5a) mRNA ratio in the developing mammalian brain. BMC Dev Biol. 2005. doi:10.1186/1471-213X-5-13.

34. Gradwohl G, Fode C, Guillemot F. Restricted expression of a novel murine atonal-related bHLH protein in undifferentiated neural precursors. Dev Biol. 1996;180:227-41. doi:10.1006/dbio.1996.0297.

35. Echelard Y, Epstein DJ, St-Jacques B, Shen L, Mohler J, McMahon JA, McMahon AP. Sonic hedgehog, a member of a family of putative signaling molecules, is implicated in the regulation of CNS polarity. Cell. 1993;75:1417-30.

36. Chan WK, Howe K, Clegg JM, Guimond SE, Price DJ, Turnbull JE, Pratt T. 2-O heparan sulfate sulfation by Hs2st Is required for Erk/Mapk signalling activation at the mid-gestational mouse telencephalic midline. PLoS One. 2015;10:e0130147.
37. Schindelin J, Arganda-Carreras I, Frise E, Kaynig V, Longair M, Pietzsch T, Preibisch S, Rueden C, Saalfeld S, Schmid B, Tinevez J-Y, White DJ, Hartenstein V, Eliceiri K, Tomancak P, Cardona A. Fiji: an open-source platform for biological-image analysis. Nat Methods. 2012;9:676-82.

38. Shimamura K, Hartigan DJ, Martinez S, Puelles L, Rubenstein JL. Longitudinal organization of the anterior neural plate and neural tube. Development. 1995;121:3923-33.

39. Robertshaw E, Matsumoto K, Lumsden A, Kiecker C. Irx3 and Pax6 establish differential competence for Shh-mediated induction of GABAergic and glutamatergic neurons of the thalamus. Proc Natl Acad Sci USA. 2013;110:E3919-26.

40. Vue TY, Aaker J, Taniguchi A, Kazemzadeh C, Skidmore JM, Martin DM, Martin JF, Treier M, Nakagawa Y. Characterization of progenitor domains in the developing mouse thalamus. J Comp Neurol. 2007;505:73-91.

41. Quinn JC, Molinek M, Martynoga BS, Zaki PA, Faedo A, Bulfone A, Hevner RF, West JD, Price DJ. Pax6 controls cerebral cortical cell number by regulating exit from the cell cycle and specifies cortical cell identity by a cell autonomous mechanism. Dev Biol. 2007;302(1-5):50-65.

42. Jones L, López-Bendito G, Gruss P, Stoykova A, Molnár Z. Pax6 is required for the normal development of the forebrain axonal connections. Development. 2002;129:5041-52.

43. Schmahl W, Knoedlseder M, Favor J, Davidson D. Defects of neuronal migration and the pathogenesis of cortical malformations are associated with small eye (Sey) in the mouse, a point mutation at the Pax-6-locus. Acta Neuropathol. 1993;86:126-35. doi:10.1007/BF00334879.

44. Stoykova A, Fritsch R, Walther C, Gruss P. Forebrain patterning defects in small eye mutant mice. Development. 1996;122:3453-65.

45. Kriegstein A, Alvarez-Buylla A. The glial nature of embryonic and adult neural stem cells. Annu Rev Neurosci. 2009;32:149-84.

46. Coutinho P, Pavlou S, Bhatia S, Chalmers KJ, Kleinjan DA, vanHeyningen V. Discovery and assessment of conserved Pax6 target genes and enhancers. Genome Res. 2011;21:1349-59.

47. Offner N, Duval N, Jamrich M, Durand B. The pro-apoptotic activity of a vertebrate bar-like homeobox gene plays a key role in patterning the Xenopus neural plate by limiting the number of chordin- and shhexpressing cells. Development. 2005;132:1807-18.

48. Kobayashi D, Kobayashi M, Matsumoto K, Ogura T, Nakafuku M, Shimamura K. Early subdivisions in the neural plate define distinct competence for inductive signals. Development. 2002;129:83-93.

49. Lim J, Choi K-W. Induction and autoregulation of the anti-proneural gene bar during retinal neurogenesis in Drosophila. Development. 2004;131:5573-80.

\section{Submit your next manuscript to BioMed Central and we will help you at every step:}

- We accept pre-submission inquiries

- Our selector tool helps you to find the most relevant journal

- We provide round the clock customer support

- Convenient online submission

- Thorough peer review

- Inclusion in PubMed and all major indexing services

- Maximum visibility for your research

Submit your manuscript at www.biomedcentral.com/submit
BioMed Central 